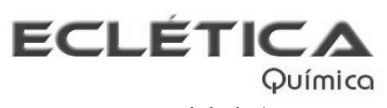

www.scielo.br/eq

Volume 30, número 1, 2005

\title{
Determinação de paracetamol em produtos farmacêuticos empregando um sistema de análise por injeção em fluxo com geração de ácido nitroso
}

\author{
W. T. Suarez, H. J. Vieira, O. Fatibello-Filho \\ Departamento de Química, Centro de Ciências Exatas e de Tecnologia, \\ Universidade Federal de São Carlos, CP 676, 13560-970, São Carlos - SP
}

\begin{abstract}
Resumo: O uso do ácido nitroso para determinação de paracetamol (acetoaminofenol) em formulações sólidas e líquidas empregando um sistema de análise por injeção em fluxo é descrito. A determinação da droga foi realizada pela reação do paracetamol com ácido nitroso gerado em linha e o produto dessa reação foi monitorado em $430 \mathrm{~nm}$ em meio alcalino. A curva analítica apresentou uma faixa de concentração de paracetamol variando entre $9,7 \times 10^{-5}$ a $6,2 \times 10^{-3} \mathrm{~mol} \mathrm{~L}^{-1}$ com um limite de detecção de $2,5 \times 10^{-5} \mathrm{~mol} \mathrm{~L}^{-1}$. Os desvios padrão relativos foram menores que $2 \%$ para soluções contendo $1,2 \times 10^{-4}$ e $2,8 \times 10^{-4} \mathrm{~mol} \mathrm{~L}^{-1}$ de paracetamol $(n=10)$ e uma freqüência de amostragem de 60 determinações por hora foram obtidos. Os resultados da determinação de paracetamol nas formulações farmacêuticas obtidas pelo procedimento em fluxo proposto estão em boa concordância com os resultados obtidos utilizando-se o procedimento da Farmacopéia Americana a um nível de confiança de 95\% e dentro de uma faixa aceitável de erro.
\end{abstract}

Palavras-chave: análise em fluxo, paracetamol, formulações farmacêuticas.

\section{Introdução}

Paracetamol (acetoaminofenol) é um analgésico antipirético derivado da fenacetina, que pode ser administrado associado com outras substâncias, como cafeína [1]. É o analgésico mais vendido no país [2]. Seu uso inadequado pode causar lesões nas células hepáticas, sendo evitado seu uso em casos de dengue, principalmente na forma hemorrágica [3]

Uma particularidade dos sistemas de análises de injeção em fluxo (FIA) que contribuiu para sua propagação como técnica analítica é a repetibilidade das operações envolvidas no sistema, como injeção, propulsão das soluções e a regulação tempo dessas operações. Com isso, sistemas de análises por injeção em fluxo permitem que sejam realizadas análises químicas, sem que a reação química tenha se completado, o que é primordial para metodologias utilizadas em bateladas. Considerando-se essas características dos sistemas em flu- xo, pode-se empregar reagentes instáveis em diversas determinações analíticas como boa precisão e exatidão, uma vez que a velocidade de decomposição do reagente pode ser controlada. O tempo de residência de um reagente no sistema de análise por injeção em fluxo é da ordem de 1 minuto, assim, o tempo de vida em que um reagente necessita ser estável não deve ser muito superior a este tempo [4,5].

Sistemas de análise por injeção em fluxo possibilitam a geração desses reagentes partindose de uma solução com maior estabilidade. No emprego de fortes agentes oxidantes, como Ag(II) e redutores, como U(III), que dificilmente são aplicados em metodologias volumétricas, pode-se partir de soluções de nitrato de prata e acetato de urânio(IV), que são convertidos pela reação com uma substância química (reagentes de Jones, para o agente redutor) ou pela reação eletroquímica (eletrodo de ouro mantido a +2 V vs ECS), para o agente oxidante [5]. 
Diversos sistemas de análises por injeção em fluxo foram desenvolvidos empregando a geração eletroquímica do reagente oxidante, a saber: $\mathrm{Ag}(\mathrm{II})$ [6,7], $\mathrm{Co}(\mathrm{III})$ [8,9], $\mathrm{Mn}(\mathrm{III})$ [10], $\mathrm{H}_{2} \mathrm{O}_{2}$ [11] e $\mathrm{Br}_{2}$ [12].

A geração de reagentes redutores pode ser realizada com o uso de um reator em fase sólida contendo, por exemplo, o reagente de Jones acoplado ao sistema de análise por injeção em fluxo. Nesses sistemas, foi possível o emprego de U(III) [13], Cr(II), V(II) [14] e Mo(III)[15], espécies altamente instáveis e de difícil aplicação analítica.

O ácido nitroso é formado pela reação de nitrito em meio ácido. Esse reagente tem sido empregado para a determinação de paracetamol [1619], salicilamida [18], oxifenobutazona [20] e metoclopramida [21]. Hererro et al. [21] desenvolveram um sistema de análise por injeção em fluxo com um reator em fase sólida contendo cádmiocuperizado, para a geração em linha de nitrito. Este sistema foi aplicado para a determinação de metoclopramida em formulações farmacêuticas. Neste sistema, uma solução de nitrato foi bombeada através do reator em fase sólida continuamente, gerando um fluxo de solução de nitrito durante todo o processo.

Vários procedimentos espectrofotométricos são descritos para determinação de paracetamol em formulações farmacêuticas, baseados na absorção de luz pelo analito na região do ultra-violeta [16] e na região do visível, após derivatização do analito com vanilina [22] ou ainda, por espectrofotometria derivativa [23]. Método volumétrico [24], voltamétrico [25], espectroeletroquímico [26], cronoamperométrico [27] e cromatográficos $[23,28]$ foram também descritos para a determinação de paracetamol em produtos farmacêuticos.

Sistemas de análise por injeção em fluxo têm sido empregados em laboratórios que empregam análises de rotina, devido à sua simplicidade, menor custo e também número elevado de determinações. Diversos sistemas de análise por injeção em fluxo têm sido descritos para determinação de paracetamol em produtos farmacêuticos, empregando deteç̧ão: espectrofotométrica [19,29-33], espectrofluorimé trica [34] e biamperométrica [35].

No presente trabalho, desenvolveu-se um sistema de análise por injeção em fluxo robusto com detecção espectrofotométrica, empregando geração “on-line” do reagente oxidante $\mathrm{HNO}_{2}$ (Equação 1, Figura 1). O ácido nitroso (1) produzido pela reação em fluxo de uma solução de nitrito de sódio com solução de $\mathrm{HCl}$, reage com o paracetamol (2) formando um produto cromogênico (3), que é instável e absorve intensamente em $410 \mathrm{~nm}$. Pela alcalinização do meio, produz uma substância altamente estável que absorve em 430 nm (substância 4 e 5), e ainda, provoca um deslocamento batocrômico, juntamente com um efeito hipercrômico [17].

\section{$\mathrm{NaNO}_{2}+\mathrm{HCl} \longrightarrow \mathrm{HNO}_{2}$ (equação 1)}<smiles>CC(=O)Nc1ccc(O)c(NS(=O)(=O)c2cc(N=O)c(N=O)cc2O)c1</smiles>

(2)

(3)

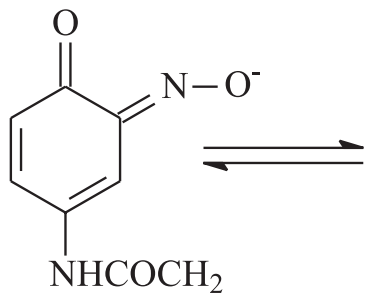

(5)

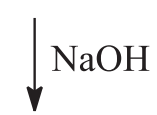<smiles>CC(=O)Nc1ccc([O-])c(N=O)c1</smiles>

(4)
Figura 1. Esquema da reação de determinação de paracetamol [17].

\section{Parte Experimental}

\section{Equipamentos}

As medidas de absorbância foram obtidas utilizando-se um espectrofotômetro Femto modelo 435 (São Paulo, Brasil) com uma cela de fluxo de vidro com um caminho óptico de $1,00 \mathrm{~cm}$, conectado a um registrador $\mathrm{x}$-t, Cole Pamer (Niles, 
IL, USA) Modelo 12020000 de dois canais. Uma bomba peristáltica Ismatec (Zurique, Suíça), modelo 7618-40 e tubos de propulsão Tygon ${ }^{\circledR}$, foram empregados para a propulsão das soluções de referência/amostras e reagente. O sistema de análise por injeção em fluxo, mostrado na Figura 2, foi construído empregando-se tubos de extensão de polietileno de 0,8 mm de diâmetro interno. Todas as soluções de amostras e de referências foram introduzidas no sistema FIA, utilizando-se um injetor-comutador [36] tipo 2-3-2 construído em nosso laboratório.

Um espectrofotômetro Hewlett-Packard (Roseville, CA, USA) modelo 8452A UV-Vis e uma cubeta de vidro de caminho óptico de $1,00 \mathrm{~cm}$ foram utilizados para obtenção das medidas espectrofotométricas do procedimento comparativo [16].

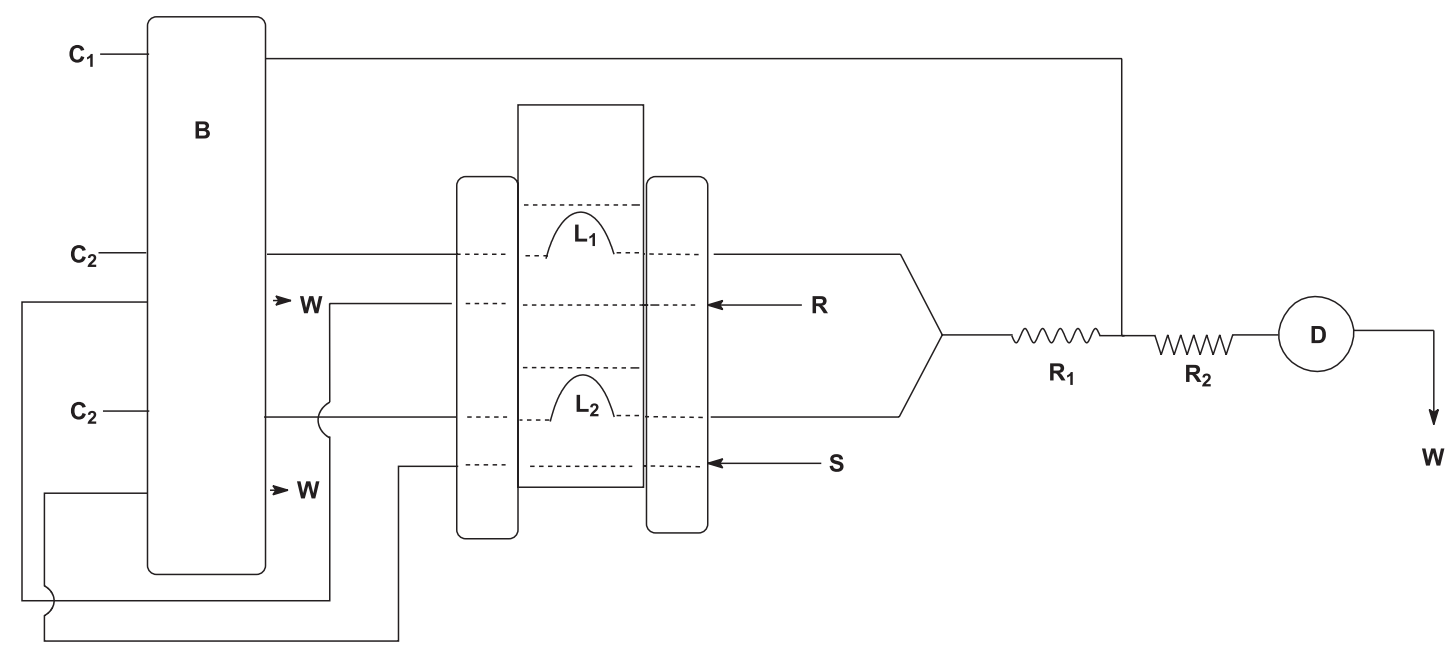

Figura 2. Sistema de análise por injeção em fluxo proposto para determinação de paracetamol em produtos farmacêuticos. R: solução de nitrito $0,6 \mathrm{~mol} \mathrm{~L}^{-1}$; $\mathbf{S}$ : solução de referência ou amostras; $\mathbf{C}_{1}$ : solução de $\mathrm{NaOH}\left(0,05 \mathrm{~mol} \mathrm{~L}^{-1}, 0,6 \mathrm{~mL} \mathrm{~min}^{-1}\right)$; $\mathbf{C}_{2}$ : solução de $\mathrm{HCl}\left(8,0 \times 10^{-3} \mathrm{~mol} \mathrm{~L}^{-1}, 2,2 \mathrm{~mL} \mathrm{~min}^{-1}\right) ; \mathbf{B}_{1}$ e $\mathbf{B}_{2}$ : bobinas reacionais de 350 e $60 \mathrm{~cm}$, respectivamente); D: detector espectrofotométrico (430 nm); W: descarte.

\section{Reagentes e Soluções}

Paracetamol (acetaminofeno), reagente de grau bioquímico (Sigma), foi utilizado para preparação de soluções de referência. Os demais reagentes utilizados foram de grau analítico e a água empregada na preparação das soluções foi proveniente de um sistema Milli-Q Millipore (Redford, MA, USA, modelo UV Plus Ultra-baixo teor de compostos orgânicos dissolvidos).

A solução estoque de paracetamol 1,5x10-2 mol L-1 foi preparada em $\mathrm{HCl}$ 8,0x10 $0^{-3} \mathrm{~mol} \mathrm{~L}^{-1}$, sendo então as soluções de referência desse fármaco nas concentrações de $9,7 \times 10^{-5}$ a $2,4 \times 10^{-3} \mathrm{~mol} \mathrm{~L}^{-1}$ preparadas anteriormente ao seu uso em $\mathrm{HCl}^{8}, 0 \times 10^{-}$ ${ }^{3} \mathrm{~mol} \mathrm{~L}^{-1}$, por diluições apropriadas da solução estoque. Prepararam-se diariamente soluções de nitrito de sódio 0,6 mol L-1 e hidróxido de sódio 0,05 mo L ${ }^{-1}$.

\section{Preparação das Amostras}

Foram realizadas determinações de paracetamol em produtos disponíveis no mercado nacional, tais como: Fluviral (DM Indústria Farmacêutica Ltda, Barueri, SP); Tylenol DC, Tylenol Extra e Tylenol Gotas (Janssen-Cilag Farmacêutica, S. J. dos Campos, SP); Excedrin (Bristol-Myers Squibb Brasil, Santo Amaro, SP); Sonridor (GlaxosmithKline Brasil Ltda, Rio de Janeiro, RJ); Dorico (Sanofi-Synthlab Ltda, Rio de Janeiro, Paracetamol (Eurofarma Laboratórios Ltda, São Paulo, SP e Paracetamol Gotas (Medley S.A Indústria Farmacêutica, Campinas, SP).

Na preparação das amostras sólidas, uma massa acuradamente pesada entre 100 e 200 mg de cada amostra foi transferida para um balão volumétrico de $100 \mathrm{~mL}$, sendo dissolvida com solução de $\mathrm{HCl} 8 \times 10^{-3} \mathrm{~mol} \mathrm{~L}^{-1}$, sendo o seu volume 
completado com a mesma solução. Um volume apropriado de cada solução de amostra foi transferido para um balão volumétrico de $100 \mathrm{~mL}$, para que a concentração da solução estivesse dentro da faixa de concentração da curva analítica.

Na preparação das amostras líquidas, alíquotas das amostras contendo paracetamol foram precisamente transferidas para um balão volumétrico de $100 \mathrm{~mL}$, sendo o volume completado com uma solução de $\mathrm{HCl}$ 8,0×10-3 $\mathrm{mol} \mathrm{L}^{-1}$. Em seguida, transferiu-se essa solução para um balão volumétrico de $50 \mathrm{~mL}$ e seu volume foi completado com a mesma solução de HCl. Dessa maneira, a concentração resultante de cada solução estava na faixa de concentração de paracetamol da curva analítica.

\section{Procedimento comparativo}

Como método comparativo, empregou-se o procedimento espectrofotométrico descrito na Farmacopéia Americana (USP-21) [16] baseado na mesma reação entre paracetamol e nitrito, em meio ácido (Figura 1), para a determinação de paracetamol nas amostras comerciais.

\section{Sistema de Injeção em Fluxo}

Um diagrama esquemático do procedimento em fluxo proposto é mostrado na Figura 2. Nesse procedimento, as soluções de referência/amostras de paracetamol e reagente foram introduzidas com o auxílio do injetor-comutador na solução transportadora ( $\mathrm{HCl} 8,0 \times 10^{-3} \mathrm{~mol} \mathrm{~L}^{-1}$, vazão de 2,2 mL $\left.\mathrm{min}^{-1}\right)$ através da alça $\mathbf{L}_{1}(75 \mathrm{~cm} ; 375 \mathrm{~mL})$. A solução do reagente nitrito de sódio $0,6 \mathrm{~mol} \mathrm{~L}^{-1}$ foi introduzida pela alça $\mathbf{L}_{2}(75 \mathrm{~cm}, 375 \mathrm{~mL})$. Ambos volumes injetados (amostra e reagente) confluem no ponto $\mathbf{C}_{\mathbf{1}}$ onde ocorre a formação do nitro-derivado do paracetamol (3, Figura 1). Após a confluência $\mathbf{C}_{2}$, ocorre a formação do respectivo íon fenolato (4-5, Figura 1), na bobina reacional $\mathbf{B}_{2}$, pela solução de $\mathrm{NaOH} 0,05 \mathrm{~mol} \mathrm{~L}^{-1}\left(0,6 \mathrm{~mL} \mathrm{~min}^{-1}\right)$, sendo essa monitorada espectrofotometricamente a $430 \mathrm{~nm}$. O sinal transiente gerado foi proporcional a concentração de paracetamol.

\section{Resultados e Discussões}

Os estudos de otimização do sistema de análise por injeção em fluxo foram realizados no modo univariado, buscando uma melhor sensibilidade e freqüência analítica.

\section{Efeito da concentração dos reagentes}

Estudou-se o efeito das concentrações de ácido clorídrico no intervalo de concentrações de $4,0 \times 10^{-3}$ a $0,02 \mathrm{~mol} \mathrm{~L}^{-1}$, hidróxido de sódio de 0,04 a $0,2 \mathrm{~mol} \mathrm{~L}^{-1}$, nitrito de sódio 0,02 a $1,0 \mathrm{~mol} \mathrm{~L}^{-1}$. O melhor sinal analítico foi obtido em solução de áci-

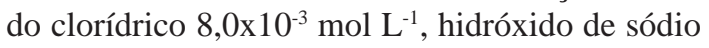
$0,05 \mathrm{~mol} \mathrm{~L}^{-1}$ e nitrito de sódio $0,6 \mathrm{~mol} \mathrm{~L}^{-1}$.

\section{Efeito do volume das alças de amostragem e do reagente}

Estudou-se o efeito do comprimento da alça de amostragem $\mathbf{L}_{1}$ e do reagente $\mathbf{L}_{2}$ de 40 a $105 \mathrm{~cm}$ (volume da amostra de 200 a $525 \mathrm{~mL}$ ) para uma solução de referência de paracetamol de $1,1 \times 10^{-3}$ mol L ${ }^{-1}$, tendo como solução transportadora o $\mathrm{HCl}$ 8,0x $10^{-3} \mathrm{~mol} \mathrm{~L}^{-1}\left(1,8 \mathrm{~mL} \mathrm{~min}^{-1}\right)$, solução de hidróxido de sódio $0,05 \mathrm{~mol} \mathrm{~L}^{-1}\left(0,6 \mathrm{~mL} \mathrm{~min}{ }^{-1}\right)$ e concentração da solução de nitrito de sódio $0,6 \mathrm{~mol} \mathrm{~L}^{-1}$. Observou-se que variando a alça de amostragem $\mathbf{L}_{1}$, sendo $\mathbf{L}_{2}$ mantida constante, o sinal analítico aumentou até o volume de $375 \mathrm{~mL}$, permanecendo constante nos volumes maiores. Dessa forma, selecionou-se esse volume de alça para os demais experimentos. Para a variação da alça do reagente $\mathbf{L}_{2}$, deixando $\mathbf{L}_{\mathbf{1}}$ com volume de $375 \mathrm{~mL}$, observou-se que o sinal analítico aumentou até o volume de $375 \mathrm{~mL}$, permanecendo constante para volumes maiores. Selecionou-se o volume de $375 \mathrm{~mL}$ como volume ótimo para demais estudos.

\section{Efeito das vazões}

Estudou-se o efeito da vazão da solução transportadora T, $\mathrm{HCl}$ 8,0x10 $0^{-3} \mathrm{~mol} \mathrm{~L}^{-1}$ de $0,7,2,2$, 2,9 e 4,2 mL min ${ }^{-1}$, nas condições descritas na Figura 2. Observou-se uma queda acentuada do sinal analítico até a vazão de 4,2 $\mathrm{mL} \mathrm{min}^{-1}$. Dessa maneira, considerando-se a freqüência analítica e a sensibilidade do procedimento em fluxo, selecionou-se a vazão de 2,2 $\mathrm{mL} \mathrm{min}^{-1}$. Estudou-se também o efeito da vazão de 0,6 a 2,9 mL minn $^{-1}$ para solução de $\mathrm{NaOH}$ 0,05 mol L-1. Observou-se uma queda da magnitude do sinal analítico com o aumento da vazão da solução de $\mathrm{NaOH}$. Dessa forma, selecionouse a vazão de $0,6 \mathrm{~mL} \mathrm{~min}{ }^{-1}$ para o sistema proposto. 
Efeito dos comprimentos da bobina reacional

Estudou-se o efeito do comprimento da bobina reacional $\mathbf{B}_{1}$ de 40, 135, 235, 350 e $422 \mathrm{~cm}$ sobre o sinal analítico, responsável pela mistura e reação entre o paracetamol e o ácido nitroso produzido. Observou-se um aumento do sinal analítico até o comprimento de $422 \mathrm{~cm}$. Dessa forma, selecionou-se o comprimento de $350 \mathrm{~cm}$ para o sistema de análise por injeção em fluxo considerando o melhor compromisso entre a freqüência analítica e a sensibilidade requerida para análise de paracetamol nas formulações farmacêuticas.

O efeito do comprimento da bobina $\mathbf{B}_{2}$, nos comprimentos de 30, 45, 60 e $100 \mathrm{~cm}$, sobre o sinal analítico foi também avaliado. Cabe lembrar, que nesse reator é formado o íon fenolato, após neutralização do excesso de ácido nitroso. Verificou-se que para os comprimentos entre 45 a 100 cm não houve perda do sinal analítico. Dessa maneira, selecionou-se assim, o comprimento de $60 \mathrm{~cm}$ como comprimento ótimo do sistema em fluxo.

Estudo da seletividade e de recuperação de paracetamol nas formulações farmacêuticas

A seletividade do procedimento de análise por injeção em fluxo proposto foi avaliada adicionando-se algumas substâncias geralmente presentes nas amostras comerciais a uma solução de refe-

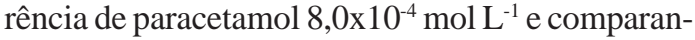
do-se o sinal analítico obtido com aquele obtido empregando uma solução de referência de mesma concentração sem adição da substância avaliada. A concentração tolerada da substância interferente foi aquela que provocou um erro igual ou menor que $5 \%$ do sinal obtido e os resultados estão mostrados na Tabela 1.

Tabela 1. Determinação de paracetamol em formulações farmacêuticas usando o método de análise por injeção em fluxo com detecção espectrofotométrica proposto e o método comparativo [16].

\begin{tabular}{cccccc}
\hline \multirow{2}{*}{ Amostras } & \multicolumn{3}{c}{ Paracetamol } & \multirow{2}{*}{$\mathrm{E}_{1}(\%)$} & $\mathrm{E}_{2}(\%)$ \\
\cline { 2 - 4 } & Valor rotulado & Comparativo & Proposto & & \\
\hline Paracetamol $^{\mathrm{a}}$ & 100,0 & $101,4 \pm 0,5$ & $100,1 \pm 0,9$ & $-1,3$ & 0,1 \\
$\begin{array}{c}\text { Paracetamol } \\
\text { Gotas }^{\mathrm{a}}\end{array}$ & 200 & $201,6 \pm 0,8$ & $201,3 \pm 0,9$ & $-0,2$ & 0,7 \\
Tylenol Gotas $^{\mathrm{a}}$ & 200 & $198,8 \pm 0,1$ & $200,3 \pm 0,3$ & 0,8 & 0,2 \\
Dorico $^{\mathrm{b}}$ & 893 & $900 \pm 3$ & $924 \pm 5$ & 2,7 & 3,5 \\
Tylenol extra $^{\mathrm{b}}$ & 697 & $635 \pm 5$ & $711 \pm 4$ & $-12,0$ & 2,0 \\
Fluviral $^{\mathrm{b}}$ & 774 & $905 \pm 3$ & $825 \pm 5$ & $-8,8$ & 6,6 \\
Tylenol DC $^{\mathrm{b}}$ & 737 & $797 \pm 3$ & $767 \pm 5$ & $-3,8$ & 4,1 \\
Excedrin $^{\mathrm{b}}$ & 766 & $836 \pm 4$ & $792 \pm 1$ & $-5,3$ & 3,4 \\
\hline
\end{tabular}

$\mathrm{n}=3$; $95 \%$ de confiança

${ }^{\mathrm{a}} \mathrm{mg} \mathrm{mL} \mathrm{m}^{-1}$

${ }^{\mathrm{b}} \mathrm{mg} \mathrm{g}^{-1}$

$\mathrm{Er}_{1}$ : erro relativo entre o procedimento proposto e o procedimento comparativo;

$\mathrm{Er}_{2}$ : erro relativo entre o procedimento proposto e o valor rotulado. 
Das substâncias investigadas, a solução de sacarose e ácido cítrico causaram uma interferência significativa no procedimento proposto. Com isso, amostras comerciais contendo ácido cítrico (produtos granulados) não puderam ser analisadas.

A recuperação de paracetamol nos produtos farmacêuticos foi avaliada adicionando-se três concentrações de solução de referência de paracetamol $\left(4,0 \times 10^{-4}, 8,1 \times 10^{-4}\right.$ e $\left.1,2 \times 10^{-3} \mathrm{~mol} \mathrm{~L}^{-1}\right)$ a oito diferentes produtos comerciais (gotas e comprimidos) e comparando-se as concentrações obtidas com as concentrações adicionadas. As recuperações variaram de 95 a 105 \%, não indicando assim um efeito de matriz acentuado nessas amostras estudadas.

\section{Curva analítica e aplicação}

A curva analítica obtida por injeções sucessivas em triplicata de seis soluções de referência de paracetamol com melhor regressão esteve no intervalo de concentração de $9,7 \times 10^{-5}$ a $6,2 \times 10^{-}$ ${ }^{3} \mathrm{~mol} \mathrm{~L}^{-1}$, preparadas em $\mathrm{HCl} 8,0 \times 10^{-3} \mathrm{~mol} \mathrm{~L}^{-1}$.
Empregando o método de mínimos quadrados, obteve-se uma equação de regressão que pode ser descrita pela equação: $\mathrm{Abs}=0,035+155,33 \mathrm{xC}-$ $9.610,77 \times C^{2} ; r^{2}=0,99989$, onde Abs é a absorbância e $C$ a concentração de paracetamol em mol L ${ }^{-1}$. O limite de detecção obtido foi de $2,5 \times 10^{-5} \mathrm{~mol} \mathrm{~L}^{-1}$ (três vezes o desvio padrão do branco/sensibilidade). A freqüência analítica obtida foi de 60 determinações por hora. Avaliou-se a repetibilidade do procedimento proposto para concentrações de $1,2 \times 10^{-4} \mathrm{~mol} \mathrm{~L}^{-1}$ e $2,8 \times 10^{-4} \mathrm{~mol} \mathrm{~L}^{-1}$ de paracetamol, obtendo-se desvios padrão relativos menores que $2 \%$ para as concentrações estudadas $(n=10)$.

A Tabela 2 apresenta uma comparação entre os teores de paracetamol encontrados nas formulações farmacêuticas analisadas utilizando-se um método comparativo descrito na Farmacopéia Americana USP-21 [16] e o procedimento de análise por injeção em fluxo proposto. Aplicando-se teste-t pareado aos resultados obtidos pelos dois métodos, verificou-se que não há diferença significativa entre os resultados obtidos a um nível de confiança de 95\% [37].

Tabela 2. Concentração tolerada de interferente na determinação de paracetamola .

\begin{tabular}{|c|c|c|}
\hline Interferente & Erro $(\%)$ & Concentração tolerada $\left(\mathrm{mol} \mathrm{L}^{-1}\right)$ \\
\hline Sacarose & $-4,5$ & $8 \times 10^{-5}$ \\
\hline Carboximetilcelulose $\mathrm{e}^{\mathrm{b}}$ & $-5,0$ & $1,5 \times 10^{-3}$ \\
\hline Manitol & $-4,8$ & $1,5 \times 10^{-3}$ \\
\hline Ácido cítrico & $-4,3$ & $8,3 \times 10^{-4}$ \\
\hline Amido $^{\mathrm{b}}$ & $-3,2$ & $4,5 \times 10^{-2}$ \\
\hline Polivinilpirrolidona ${ }^{b}$ & $-2,0$ & $1,7 \times 10^{-2}$ \\
\hline Acessulfame-K & $-3,8$ & $2,3 \times 10^{-2}$ \\
\hline Cafeína & $+1,1$ & $6,1 \times 10^{-4}$ \\
\hline Sacarina & $+1,0$ & $1,5 \times 10^{-3}$ \\
\hline Ácido ascórbico & $-4,2$ & $1,8 \times 10^{-4}$ \\
\hline
\end{tabular}

${ }^{a}$ concentração de paracetamol: $8 \times 10^{-4} \mathrm{~mol} \mathrm{~L}^{-1}$;

ь $\%(\mathrm{~m} / \mathrm{v})$. 


\section{Conclusões}

O procedimento de análise por injeção em fluxo com detecção espectrofotométrica proposto é um sistema mecanizado simples, que se apresentou preciso e com sensibilidade para determinação de paracetamol em formulações farmacêuticas. É um sistema em fluxo de maior robustez quando comparado com sistemas cromatográficos e de fluxo como aqueles propostos anteriormente $[19,23,28]$. Apresentou uma curva analítica com uma maior intervalo de concentração quando comparada com outros sistemas de análises por injeção em fluxo para de- terminação de paracetamol [19,30,32,33] e ainda, apresentou um menor consumo de nitrito por determinação, comparado com procedimentos em batelada [16-19].

\section{Agradecimentos}

Os autores agradecem ao CNPq, à CAPES e à FAPESP pelo suporte financeiro e ao CNPq pela bolsa de mestrado de H. J. V e W. T. S.

Recebido em: 01/03/2004

Aceito em: 03/09/2004

W. T. Suarez, H. J. Vieira, O. Fatibello-Filho. Determination of paracetamol in pharmaceutical products using a flow injection analysis system with generation of nitrous acid.

\begin{abstract}
The use of nitrous acid $\left(\mathrm{HNO}_{2}\right)$ for determination of paracetamol (acetoaminophen) in liquid and solid formulations employing flow injection system is described. The determination of this drug was carried out by reacting paracetamol with nitrous acid generated in-line and the product yielded was measured at $430 \mathrm{~nm}$ in alkaline medium. The analytical curve ranged from $9.7 \times 10^{-5}$ to $6.2 \times 10^{-3} \mathrm{~mol} \mathrm{~L}^{-1}$ of paracetamol, with a detection limit of $2.5 \times 10^{-5} \mathrm{~mol} \mathrm{~L}^{-1}$. The relative standard deviations (RSDs) were smaller than $2 \%$ for solutions containing $1.2 \times 10^{-4}$ and $2.8 \times 10^{-4} \mathrm{~mol} \mathrm{~L}^{-1}$ of paracetamol $(\mathrm{n}=10)$ and 60 determinations per hour were obtained. The results of determination of paracetamol in pharmaceutical formulations obtained by the proposed FI procedure were in good agreement with those obtained using theUSP procedure at a confidence level of $95 \%$ and within an acceptable range of error.
\end{abstract}

Keywords: Flow injection spectrophotometry; nitrous acid; paracetamol.

\title{
Referências
}

[1] A. Korolkovas, J. H. Burckhalter, Química Farmacêutica, Guanabara Dois, Rio de Janeiro, 1982, p.159-197.

[2] Site: http://www.sobravime.org.br/disc_dipirona.html, visitado em 14/08/2004.

[3] Site: http://www.anvisa.gov.br/farmacovigilancia/informes/ 2002/informe_2.htm, visitado em 20/08/2004.

[4] G. Den Boef, R. C. Schothorst, Anal. Chim. Acta 180 (1986) 1-3.

[5] G. Den Boef, Anal. Chim. Acta 216 (1986) 289.

[6] X. B. Li, Z. J. Zhang, M. Wu, Anal. Chim. Acta 432 (2) (2001) 311.

[7] R. C. Schothorst, G. Den Boef, Anal. Chim. Acta 169 (1985) 99.

[8] B.X. Li, Z. J. Zhang, M. L. Wu, Mikrochim Acta 134 (34) (2000) 223.

[9] B. X. Li, Z. J. Zhang, M. Wu, Talanta 51 (3) (2000) 515. [10] R. C. Schothorst, O. O. SChmitz, G. Den Boeff, Anal. Chim. Acta 179 (1986) 299.

[11] C. Zhang, J. Huang, Z. Zhang, M. Aizawa, Anal. Chim. Acta 374 (1) (1998) 105.

[12] X. W. Zheng, M. Yang, Z. J. Zhang, Anal. Lett. 32
(1999) 3013.

[13] R. C. Schothorst, M.; Van Son, G. Den Boef, Anal. Chim. Acta 162 (1984) 1.

[14] R. C. Schothorst, G. Den Boef, Anal. Chim. Acta 175 (1985) 305.

[15] W. Th. Kok, D. T. Thuy, T. V. Nghi, G. Den Boef, Anal. Chim. Acta 200 (1987) 533.

[16] United States Pharmacopeia National Formulary XXI, United Pharmacopeial Convention, Inc., Rockville, MD, 1985, p. 11-15.

[17] S. F. Belal, M. A. H.; El Sayed, A.; El Walily, H. Abdine, Analyst 104 (1979) 919.

[18] M. I. Walash, A. M. El-Brashy, M. A. Sultan, Mikrochim. Acta 113 (1994) 113.

[19] M. Knochen, J. Giglio, B. F. Reis, J. Pharm. Biomed. Anal. 33 (2003) 191.

[20] A. Abou Ouf, M. I. Walash, S. M. Hassan, S. M. ElSayed, Analyst 105 (1980) 169.

[21] M. R. Herrero, A. M. Romero, J. Martínez-Calatayud, Talanta 47 (1) (1998) 223.

[22] F. M. Plakogrannis, A. M. Saad, J. Pharm. Sci. 64 (9) 
(1975) 1547.

[23] E. Dinç, G. Kökdil, F. Onur, J. Pharm. Biomed. Anal. 26 (5-6) (2001) 769.

[24] European Pharmacopoeia, Strasbourg: Council of Europe, 1997, p. 1032.

[25] J. M. Zen, Y.S. Ting, Anal. Chim. Acta 342 (1997) 175. [26] Y. Z. Fang, D. Long, J. Ye, Anal. Chim. Acta 342 (1997) 13.

[27] O. Fatibello-Filho, K. O.Lupetti, I. C.Vieira, Talanta 55 (4) (2001) 685.

[28] J. L. Perez, M. A. Bello, Talanta 48 (5) (1999) 1199. [29] J. Martínez-Calatayud, Anal. Lett. 19 (19-20) (1986) 2023. [30] A. R. Medina, M. L. F. de Córdova, A. M. Díaz, Anal. Chim. Acta 394 (1999) 149.

[31] J. Martínez-Calatayud, C. G. Benito, Anal. Chim. Acta
231 (1990) 259.

[32] C. Aniceto, O. Fatibello-Filho, Quim. Nova 25 (3) (2002) 387.

[33] A. C. Criado, S. Cárdenas, M. Gallego, M.Válcarcel, Talanta 53 (2000) 417.

[34] Sh. M. Al-Ghannam, A. M. El-Brashy, B. S. Al-Farhan, Farmaco 57 (8) (2002) 625.

[35] A. M. Galvez, J. V. García Mateo, J. Martínez-Calatayud, Anal. Chim. Acta 396 (2-3) (1999) 161.

[36] B. F. Reis, H. Bergamin-Filho, Quim. Nova 16 (1993) 570 .

[37] J. C. Miller, J. N. Miller, Estadística para Química Analítica, segunda Editión, Addison-Wesley Iberoamericana, S.A: Wilmington, E.U.A, p. 42. 